\title{
Risk Perceptions of Acquiring Type 2 Diabetes Mellitus Among College Students
}

\author{
Seth Simmons \\ University of Mississippi \\ Mary Allison Ford-Wade \\ University of Mississippi \\ Milorad Novicevic \\ University of Mississippi \\ Ifeoluwa Tobi Popoola \\ McMurry University
}

The rapidly increasing number of Type II diabetes mellitus cases among adolescents highlights the need to understand the risk perceptions of adolescents towards this disease. Our study addresses this need by examining students' risk perceptions of acquiring Type II diabetes mellitus. Upon unpacking the conceptualization of students' risk perceptions and reviewing the relevant literature, we develop and test empirically a comprehensive model incorporating personal, public, and environmental risk factors influencing students' risk perceptions of acquiring Type II diabetes mellitus. Our findings support the significant impact of these factors.

\section{INTRODUCTION}

Type II diabetes mellitus is a part of a group of metabolic disorders involving high blood glucose levels over a prolonged period of time (Alawneh, Yasin, Khirfan et al., 2016; National Institute of Diabetes and Digestive and Kidney Diseases, 2017). While it is one of the most commonly pervasive chronic diseases in the world, national awareness of Type 2 diabetes mellitus is imperative because it is the seventh-leading cause of death in the United States (CDC - Centers for Disease Control and Prevention, 2017). Moreover, this disease affects over 30.3 million Americans or $9.4 \%$ of the population with 23.1 million people diagnosed with diabetes. When it is undiagnosed, the disease results in chronic health consequences such as vision loss, kidney failure, nerve damage, and high blood pressure (National Institute of Diabetes and Digestive and Kidney Diseases, 2017). Specifically, CDC pointed out in its 2017 national diabetes statistics report that 30.2 million adults at least 18 years or older (i.e. $12.2 \%$ of all U.S. adults) had diabetes, of which 7.2 million (i.e. 23.8\%) were either unaware the possessed the disease or failed to report having diabetes (p.2). This report is based on results from three separate national data sources: 1) the 2011-2014 National Health and Nutrition Examination Survey (NHANES) data, 2) the 
2013-2015 National Health Interview Survey (NHIS) data, and 3) the 2010-2015 U.S. Census Bureau resident population data.

As a leading cause of morbidity and mortality, T2DM will likely "become an even greater public health concern if the current rates of obesity continue... as 3600 youths are newly diagnosed with T2D annually" (Amuta et al., 2016, p.786). Diabetes mellitus takes two forms: 1) Type 1 diabetes mellitus (abbreviated as T1DM) and, 2) Type 2 diabetes mellitus (abbreviated as T2DM). T1DM is an autoimmune disorder involving the body's inadequate production of the insulin hormone that is critical to lowering high blood glucose levels. T2DM, on the other hand, is an autoimmune disorder that limits target insulin cells from responding to the body's insulin distribution, thus resulting in the buildup of glucose in the blood and a reduction of insulin cells required to generate energy (Mayo Clinic, 2017). Majority of extant diabetes research is focused on T2DM because $90-95 \%$ of individuals affected with diabetes are diagnosed with T2DM. Therefore, in this study, we examine the risk perceptions of T2DM among students, a highly vulnerable group.

The purpose of this paper is to develop and empirically test a comprehensive model of students' risk perceptions of acquiring diabetes. The paper is organized in the following way. First, we provide a conceptualization of students' risk perceptions of acquiring diabetes based on the review of the relevant related literature. Second, we develop a comprehensive model that incorporates personal, public and environmental risk factors proposing that they influence the students' risk perceptions. Third, we describe the method that we applied to test our proposed model. Fourth, we provide the results and discuss the findings of our statistical analysis. In conclusion, we outline practical implications and limitations of this study.

\section{Theoretical Foundation}

Social cognitive theory (Bandura, 1977; Bandura, 1986, 1991, 1999) posits that individuals in general and adolescents in particular influenced by the cues that they face across situations not only experience motivating drive to act but also are influenced by their perceptions of the consequences of their actions (Kreps \& Monin, 2011; Font, Garay, \& Jones, 2016). In the context of college students' perceptions of acquiring T2DM, these perceptions are likely to be influenced additionally by their individual characteristics and learning abilities, interactions with other adolescents within the school environment, and their individual personalities. These factors are constantly in interplay influencing individual perceptions of students (Font et al., 2016). Thus, social cognitive theory posits that the interplay between college students' past behaviors, individual personal characteristics, and the social context or environment in which they interact within may influence their T2DM risk perceptions.

\section{Conceptualization of Students' Risk Perceptions of Acquiring Diabetes}

At the end of the 20th century, T2DM accounted for less than $3 \%$ of new-onset cases of diabetes in adolescents. However, by the end of the second decade of the 21 st century, the increasing diabetic symptoms diagnosed became one of the most pressing national health concerns among adolescents and children (D'adamo \& Caprio, 2011). Therefore, T2DM no longer fits the label of adult-onset diabetes as more children, adolescents, and students are diagnosed with the diabetic disorder, most likely due to the increasing prevalence of early obesity (Mayo Clinic, 2017). Specifically, in 2015, 132,000 children and adolescents under 18 years (i.e. representing $0.18 \%$ of the adolescent population in the United States) or 193,000 children and adolescents under 20 years (representing $0.24 \%$ of U.S. population) were diagnosed with T2DM (CDC, 2017). The CDC in 2017 also pointed out that T2DM is most prevalent among American Indians/Alaska Natives (15.1\%), followed by non-Hispanic blacks (12.7\%), Hispanics (12.1\%), and then Asian, Non-Hispanic (8.0\%) (p. 3, 13).

The 2017 National Diabetes Statistics report showed that 84.1 million Americans were pre-diabetic in 2015 with more men (36.6\%) than women (29.3\%) having prediabetes (CDC, 2017). Pre-diabetes is a condition manifested with the people that do not show diabetic symptoms of abnormally high blood glucose levels but are not officially diagnosed with diabetes. These pre-diabetic patients are typically more likely to become diabetic due to inherent non-modifiable factors such as genetic history, race, 
ethnicity, and age (Valdez et al., 2007). In addition, people with both mother and father having diabetes are two to four times likely to develop diabetes (Alcolado \& Alcolado, 1991; K Papazafiropoulou et al., 2017; Geetha et al., 2017). This is also the case with the individuals with a minimum of one first-degree relative from the same maternal or paternal ancestry having T2DM and two first-degree relatives having T2DM (Vornanen et al., 2016) who are respectively 2.3 and 5.5 times more likely to be diagnosed with T2DM (Claassen, Henneman, \& Janssens, 2010; Amuta et al., 2016). The high prevalence of T2DM among family members may be due to family members sharing similar biological traits, behaviors, norms, and cultures (Claassen et al., 2010).

The T2DM-modifiable or "self-managed" behaviors such as diet and exercise, are the common factors that are likely to improve people's health by decreasing the likelihood to be diagnosed with diabetes. As the impact of the engagement in dieting depends on the level of education, more individuals without a high school degree are diagnosed with diabetes $(12.6 \%)$ than those with either a high school degree $(9.5 \%)$ or those with post-high school education $(7.2 \%)$. In addition, a person's perceived risk, as perceived susceptibility or vulnerability to diabetes, is a critical determinant whether individuals will take steps to prevent or treat their diabetes symptoms. The perceived risk of acquiring diabetes is important to know because it indicates whether the person is aware enough of the necessity to take action to control his or her health problems (Rouyard, Kent, Baskerville, Leal, \& Gray 2017). On the one hand, the more individuals underestimate the severity of their diabetic risks, the less unlikely it is that they will engage in behaviors that could improve their health. On the other hand, when people overestimate their risks to developing diabetes, they experience undue stress (Janz \& Becker, 1984).

A common misconception of the risk perception to acquire diabetes is that it can be assessed outright as an objective probability that is always measurable accurately and assessable outright. In reality, risk perception is complex phenomenon of multiple types. Specifically, Rouyard, Kent, Baskerville, Leal, and Gray (2017) classify risk perceptions into three types: 1) absolute risk perception, 2) comparative risk perception, and 3) conditional risk perception. Absolute risk perception reflects the difference between one's perceived and actual risk. Comparative risk perception involves the comparison between one's perceived risk and another person's perceived risk. Lastly, conditional risk perception reflects a perception that the downward outcome of acquiring diabetes will occur if a certain behavior is adopted over a period of time.

Optimistic bias is a key issue limiting the effective measurement of all three types of risk perception. Optimistic bias, which refers to the "illusion of invulnerability" reflects individual tendency to underestimate the likelihood to experience negative outcomes (such as acquiring diabetes) while overestimating the likelihood to experience positive outcomes. These underestimations of exposure to harming events and overestimations of exposure to positive events are common among younger individuals who are unlikely to fully understand long-term consequences of their current health-related behaviors. Moreover, extant research studies provide significant evidence that individual risk perception "often go awry" (Waters et al., 2013) due to an overestimation of the risk of developing a disease such as T2DM because they may develop anxiety as well as the unwillingness and inability to take preemptive preventative measures (Rouyard et al.,2017). In the subsequent section, we provide the literature review of research on risk perceptions of T2DM among adolescents.

\section{Perceived Risk of Acquiring Diabetes}

Research on risk perceptions of acquiring T2DM is in a nascent phase (Bloomgarden, 2004; Amuta, Jacobs, Barry, Popoola, \& Crosslin, 2016). The scarcity of this research is also evidenced by the 2016 study published by the researchers at the Health Economics Research Centre, University of Oxford (HERC). This study is a systematic review of 18 studies exploring qualitative and quantitative studies on risk perceptions for diabetes-related complications. Twelve of these studies focused on T2DM cardiovascular risks, while the remaining six studies focused on microvascular risks or complications related to the eyes, hands, and the feet. The studies addressed 23 "outcomes" classified into five risk categories: absolute accuracy, comparative risk, risk questionnaires, risk perception scores, and complementary evidence. 
The category of Rouyard et al.'s (2017) studies on absolute accuracy - referred to as the average of individual perceived absolute risks - yielded mixed results because people tend to overstate the plausibility of having a heart attack and stroke, and to underestimate the risks of diabetes-related eye complications. The studies revealed that optimistic bias outcomes were higher among minority ethnic groups, as well as among those with lower educational levels. Interestingly, Homko et. al (2018), showed that women tend to exhibit higher perceived risk for cardiovascular disease compared to men. Overall, qualitative studies involving focus groups and semi-structured interviews, revealed that a large proportion of the population, including $70 \%$ of respondents with T2DM in a single large study, were unaware that they were at risk for cardiovascular disease.

The researchers at the Diabetes Research and Training Center, Albert Einstein College of Medicine designed a study to explore the comparative risk judgments for diabetes of physicians in northeastern United States and Canada (Walker, Mertz, Kalten, \& Flynn, 2003; Walker et al., 2007). Highlighting the need to overcome latent communication barriers between doctors and patients, this research study underscored the need to better understand the attitudes and perceptions of professionals and neophytes related to developing diabetes. In this study, a total of 535 non-diabetic individuals were surveyed using the revised Risk Perception Survey for Developing Diabetes (RPS-DD) and American Diabetes Association Diabetes Risk Test instruments containing 53 items. The population was divided into two groups: 1) participants with high risks of developing T2DM, and 2) participants with low risks of developing diabetes. Participants in the high-risk group exhibited a tendency to worry about developing diabetes, as well as a lower sense of personal control and a greater overall perceived risk of exposure to multiple diseases. Most participants in the low risk group in terms of developing diabetes exhibited higher levels of optimistic bias towards developing diabetes compared to $50 \%$ of participants in the group with higher risks of developing diabetes. Overall, these findings, supported by an earlier pilot study of nonphysicians, provide evidence of optimistic bias.

\section{General Factors Related to Diabetes Risk Perception}

To examine factors related to risk perception, Fischetti (2015) conducted a study testing the relationships between perceived T2DM risk and diet and exercise regimens. She surveyed a group of high school students and Boy Scout troop members in New York City, excluding those with a family history of T1DM or T2DM. Fischetti's study of 35 male and 45 female students with ages ranging from 13-18 years is one of the few diabetes studies that specifically focused on adolescents' risk perceptions of T2DM. Her study required four main survey instruments: 1) the Godin (2011) Leisure-Time Exercise Questionnaire used to evaluate weekly physical activity; 2) the revised 14 item Risk Perception Survey for Developing Diabetes (Walker et al., 2003); 3) optimistic bias survey developed to measure worry, personal control, dread, and unknown risks; and 4) participants' diary used to jot recollections of two dietary intakes over a 24-hour period.

Moreover, Fischetti's (2015) study yielded three general findings. First, perceived T2DM risk and dietary intake were not correlated; whereas there was a significant negative inverse relationship between dread and fat intake on one hand and a significant positive relationship between dread and carbohydrate intake on the other hand. Second, exercising contributed to a significant inverse relationship between perceived risk of T2DM and strenuous physical activity. Third, teens tend to have a false understanding of the role of carbohydrates in their diet and those who exercised more, even in moderate amounts, were less likely to perceive a risk of having T2DM. These significant findings have two limitations. First, several reliability coefficients were not high enough to meet research standard levels, most likely due to the novelty of her survey instrument. Second, the small sample size signaled that homogeneity issues were inherently imbedded in her results.

In another study, Sealey-Potts and Reyes-Valesquez (2014) surveyed 660 university students following the Center for Disease Control's diabetes screening guidelines. The study, consisting of 63.2\% female and $36.8 \%$ male students, compared participants' actual T2DM risk level to their perceived T2DM risk level. The survey consisted of 27 items measuring comparative and actual risk perceptions. The actual risk perceptions, scored on a total points scale, measured risk perceptions of: parents with diabetes 
(1 point), sister of brother with diabetes ( 1 point), overweight/obese classification ( 5 points), age less than 65 years with three or less week days of physical activity (5 points), and ethnic minority group (1 point). Scores of 3-8 indicate low risk of T2DM and score of 9 or more indicate high risk of T2DM.

Similarly, Sealey-Potts and Reyes-Valesquez's (2014) study measured participants' demographic data (such as age, gender, weight, height, and body mass index) and polled their daily fruit and vegetable intake. Results supported the hypothesis that participants in the high-risk group were at "some" or "great" risk for developing T2DM. Specifically, participants in the high-risk group scored higher on their actual risk perception compared to participants in the low risk group. While the mean score of 6.63 indicates a low risk for T2DM, observed scores of the high and low risk groups were 10.14 and 3.34 respectively. Fifty percent of the participants were unsure of their personal risks due to lifestyle behaviors whereas twenty-six percent were unsure of risks related to family history. Over $40 \%$ participants were classified as obese. These findings support the presence of optimistic bias because most of the participants were unaware of their personal risks and were also more likely to underestimate their risk of developing diabetes.

\section{Research Questions Guiding this Study}

Our literature review indicates that past research on adolescents' risk perceptions of acquiring T2DM was fragmented. Therefore, there is a pressing need for developing and testing a comprehensive model of adolescents' perceptions of their health risks towards diabetes focusing on the personal, public and environmental drivers of diabetes risk perceptions. The increasing rates of diabetes among younger age groups makes it imperative to examine the relationships among these drivers and their potential significant effect on adolescents' perceived health risk of acquiring diabetes. Our proposed comprehensive model, which incorporates these drivers (see Figure 1), is proposed to address the following four research questions:

1. What external and/or environmental factors influence adolescent risk perception?

2. What internal and/or personal factors influence adolescent risk perception?

3. What effects do perceptions of the general public have on diabetes risk perception?

4. What is the relationship between external, internal, and general public factors; and how do these factors effect overall risk perception of T2DM?

\section{FIGURE 1 \\ HYPOTHESIZED MODERATED MEDIATION MODEL OF RISK PERCEPTIONS OF TYPE II DIABETES MELLITUS}

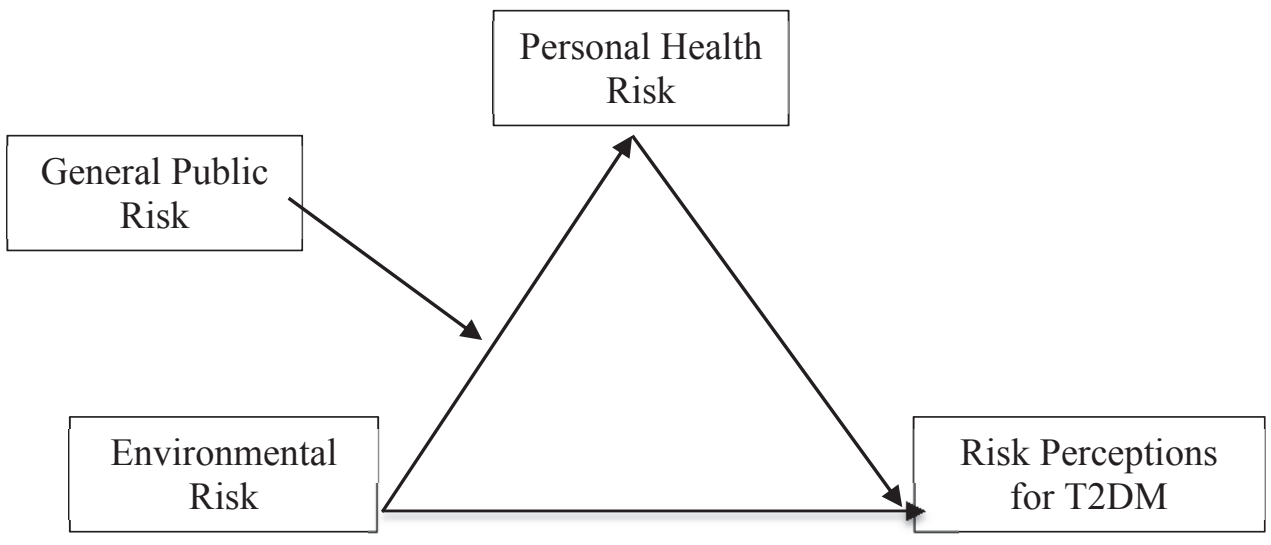




\section{Description of Proposed Model}

Out proposed model is a moderated mediation model. A moderated mediation model describes the indirect effect an independent variable (x) has on (y) through a mediator (m), moderated by another variable (w). Depending on the value of the "w," results of x's effect on y can vary drastically (Preacher, Rucker \& Hayes, 2007). Applying this model to our study, we use it to hypothesize the effects of environmental public and personal risk perceptions (ER) on one's risk perceptions of T2DM (RPT). The control variables include the number of weekly exercises (Godin, 2011), gender, race, employment status, and class level. In the subsequent section, we describe the method that we use to test empirically our proposed model.

\section{METHOD}

\section{Participants and Procedure}

To test the proposed model, we employed a cross-sectional survey design. The survey items were adapted from the Albert Einstein College of Medicine's Risk Perception Survey for Developing Diabetes (RPS-DD) instrument and items from Godin's (2011) Leisure-Time Exercise Questionnaire. Also, the authors emailed a survey link to 5000 undergraduate students at a University located in Southeastern United States and collected participants' demographic data using Qualtrics, an online survey platform (Mei \& Brown, 2018). Bi-weekly reminders were sent over a two-week period to encourage participants to complete the survey. See Appendix A for survey instruments.

\section{Statistical Analyses}

The data was analyzed with IBM's (2017) Statistical Package for the Social Sciences (SPSS, version 25) and Andrew Hayes' (2018) PROCESS (version 3.0) statistical software. We assessed the mediating effect of personal health risk (PHR) on the relationship between environmental risk (ER) and risk perceptions for T2DM (RPT). Moreover, the authors assessed the moderating effect of general public risk on the relationship between ER and PHR as well as the indirect moderating effect of the moderator on RPT. After ensuring the dataset was cleaned to eliminate biased responses from the dataset, the authors conducted an exploratory factor analysis to ensure that the Cronbach coefficient alpha reliability $(\alpha)$ factor loadings range for each survey instrument meets the acceptable criteria published in extant research. Cronbach alpha is a general measure of reliability or internal consistency of a scale (Yang \& Green, 2011). And as presented below in Table 1 below, the Cronbach coefficient alpha reliability for each survey instrument exceeds the minimum 0.70 threshold (Alawneh et al., 2016).

TABLE 1

\section{RELIABILITY STATISTICS FOR SELECTED MEASURES}

\begin{tabular}{|l|c|}
\hline Reliability & Cronbach alpha \\
\hline & \\
\hline RPT & 0.778 \\
\hline ER & 0.852 \\
\hline PHR & 0.773 \\
\hline GPR & 0.728 \\
\hline
\end{tabular}




\section{Measures}

a. Risk Perception (RPT): These measures overall T2DM risk perceptions, revised version of the Risk Perception Survey for Developing Diabetes survey (RPS-DD) (Diabetes Research and Training Center, Albert Einstein College of Medicine, 2003) was used. The 4-point Likert-scale items was used for measuring participants' feelings of control, worry, and susceptibility of getting the disease ranged from $1=$ Almost No Risk to $4=$ High Risk.

b. Public Health Risk (PHR): This is an individual rating of the likelihood to be at risk for a disease (such as arthritis, heart disease, cancer, and high blood pressure), based on one's own personal characteristics, family history and medical history. The 4-point Likert scale items adapted from the RPS-DD ranged from $1=$ Almost No Risk to $4=$ High Risk.

c. Environmental Risk (ER): The revised version of the RPS-DD instrument was used to measure perceptions of external threats (e.g. second-hand smoke, driving/riding in an automobile, and illegal drugs) that may impact a person's health. The four-point Likert scale ranged from $1=$ Almost No Risk to $4=$ High Risk.

d. General Public Risk (GPR): This is a 4-point Likert-type scale from $1=$ Decreases the Risk to $4=$ Don't know measure perceptions of demographic and lifestyle characteristics' (e.g. age, ethnicity, diet, exercise, and family history) effect on risk of developing T2DM.

e. Control Variables: Based on extant literature (e.g. Persons, 2009; Amuta et al., 2016; Popoola et al., 2017) we controlled for the number of participants' weekly exercises (Godin, 2011) using a 3point Likert scale ranged from $1=1-3$ times per week to $3=5$ or more times per week. Also, we controlled for participants' gender, race, employment status, and class level.

\section{Sample Description}

A total of 155 responses (i.e. 47.69\%) were usable from 325 completed questionnaires. Eliminated responses included those from participants that spent excessive time to complete the survey, participants that spend insufficient time completing the survey, and participants that partially completed the survey questions. In the remaining study responses, $70.3 \%$ participants were female and $29.7 \%$ were male. All participants (i.e. $96.1 \%$ ) were between 18 years and 24 years, except for six participants who were older than 25 years. In particular, the average of the six participants is 33.83 years. With regards to the ethnicity of participants, $83.9 \%$ are white, $9 \%$ were Black, 32.2\% Asians, $2.6 \%$ Hispanic, and $1.3 \%$ Other. The ethnic demography is a general reflection of the city's population. Lastly, about one-third of respondents (i.e. $32.9 \%$ ) had at least one family member with a history of diabetes.

\section{Correlation Results}

The correlation, descriptive statistics, and reliabilities of selected measures are presented in Table 2. As hypothesized, Environmental Risk is positively correlated with Personal Health Risk $(\mathrm{r}=.259, \mathrm{p}<.01)$ at a $99 \%$ confidence interval whereas, General Public Risk is positively correlated with Personal Health Risk $(\mathrm{r}=.173, \mathrm{p}<.05)$ at a $95 \%$ confidence interval. Lastly, there was no significant direct effect of Environmental Risk (ER) on Risk Perception for T2DM (RPT) $(r=-0.045, \mathrm{p}>0.05)$, there still exists a conditional indirect effect on RPT through PHR and moderated by GPR. 
TABLE 2

CORRELATION, MEAN, AND STANDARD DEVIATION OF STUDY VARIABLES

\begin{tabular}{|l|r|r|r|r|r|r|r|r|r|c|}
\hline & RPT & PHR & GPR & ER & Gender & Race & $\begin{array}{c}\text { Full/Part- } \\
\text { Time }\end{array}$ & Exer & Mean & SD \\
\hline RPT & 1 & & & & & & & & 2.41 & 0.986 \\
\hline PHR & 0.139 & 1 & & & & & & & 1.36 & 0.478 \\
\hline GPR & 0.005 & $\mathbf{0 . 1 7 3}$ & 1 & & & & & & 1.77 & 0.426 \\
\hline ER & -0.045 & $\mathbf{0 . 2 5 9 * *}$ & 0.023 & 1 & & & & & 2.19 & 0.782 \\
\hline $\begin{array}{l}\text { Gender } \\
\text { Sub. }\end{array}$ & -0.027 & 0.130 & 0.043 & 0.059 & 1 & & & & 1.30 & 0.458 \\
\hline Race & -0.026 & -0.016 & -0.053 & -0.024 & -0.038 & 1 & & & 1.28 & 0.762 \\
\hline $\begin{array}{l}\text { Full/Part- } \\
\text { Time }\end{array}$ & 0.069 & 0.006 & 0.070 & 0.012 & 0.016 & 0.013 & & & 1.04 & 0.194 \\
\hline Exercise & $\mathbf{- 0 . 2 0 5}$ & 0.133 & 0.002 & $\mathbf{0 . 1 6 6}$ & $\mathbf{0 . 1 5 9}$ & -0.037 & 0.011 & 1 & 1.30 & 0.539 \\
\hline
\end{tabular}

\section{Analysis of Direct and Indirect Effect}

Results indicate that the relationship between ER and PHR is significant at the $95 \%$ Confidence Interval (CI) level. Specifically, ER not only predicted PHR $(\beta=.150, \mathrm{p}=.002)$ but also, the interaction effect of ER and GPR on PHR is significant $(\beta=.330, p=.01)$ at the $95 \%$ CI level. Moreover, the results indicated that PHR significantly predicted RPT $(\beta=.361, \mathrm{p}=.036)$ while ER did not predict RPT $(\beta=-$ $.077, \mathrm{p}=.462)$. Lastly, results show that PHR mediated the relationship between ER and RPT $(\beta=.072)$. Results are presented in Table 4 and Figure 2.

\section{FIGURE 2 \\ ESTIMATES OF MODERATED MEDIATION MODEL}

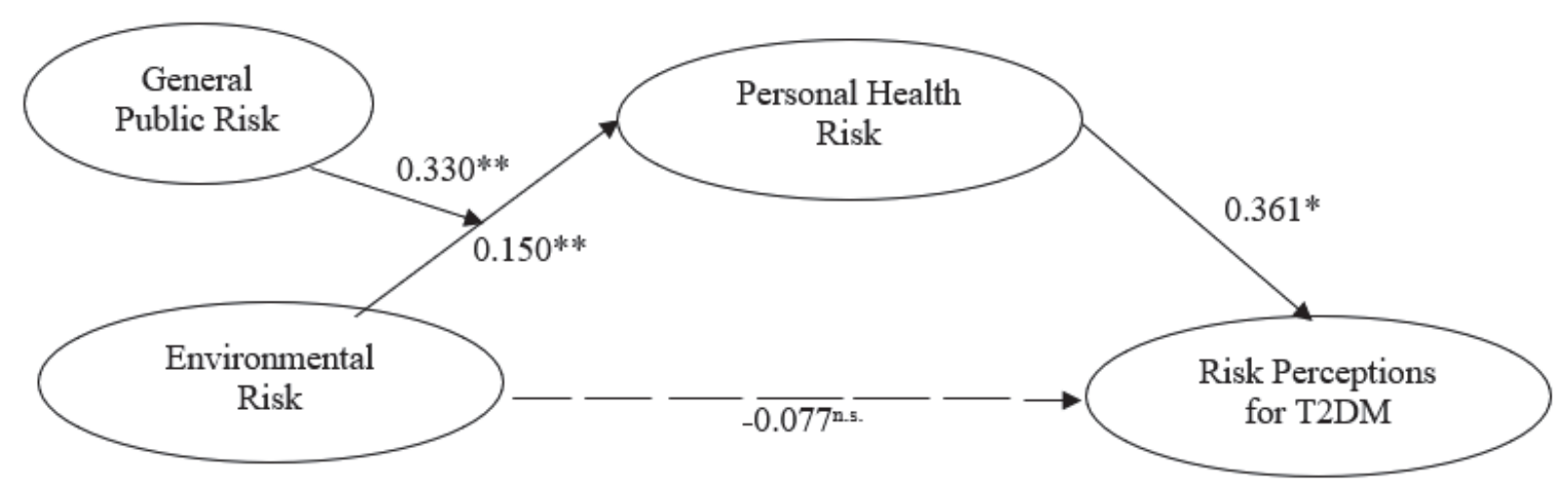

${ }^{n . s .} p=$ not significant; $* p<0.05 ; * * p<0.01$

\section{Moderated Mediation Analysis}

After determining that significant mediation exists between our key variables, the authors examined the mean centered effect of the moderator, GPR. Results presented in Table 4a show that the conditional indirect effects, based on $95 \%$ CI of ER (i.e. the focal predictor) on RPT at values of the moderator is significant at the mean level (i.e. 50th percentile) and at 1 standard deviation below the mean (i.e. at the $84^{\text {th }}$ percentile). In other words, at a one standard deviation below the mean, the moderating effect of GPR 
is not statistically insignificant, because zero lies between the lower and upper level bounds of the CI. However, when GPR is at its mean and one standard deviation above the mean, there is a significant effect of the moderator, GPR.

TABLE 3

PATH ESTIMATES FOR DIRECT EFFECT ON RPT AND PHR; AND INTERACTION EFFECT OF ER AND GPR ON PHR

\begin{tabular}{lllll}
\hline \multicolumn{1}{c}{ Estimates } & $\mathrm{SE}$ & $\mathrm{t}$ & $95 \%$ CI (bootstrap) & $\mathrm{P}(>|\mathrm{t}|)$ \\
\hline Direct Effects & & & & \\
$\mathrm{ER} \longrightarrow \mathrm{RPT}-0.077$ & 0.104 & -0.737 & {$[-0.272,0.147]$} & 0.462 \\
$\mathrm{PHR} \longrightarrow$ RPT 0.361 & 0.171 & 2.117 & {$[0.077,0.622]$} & 0.036 \\
$\mathrm{ER} \longrightarrow$ PHR 0.150 & 0.047 & 3.173 & {$[0.056,0.249]$} & 0.002 \\
GPR $\longrightarrow$ PHR 0.121 & 0.089 & 1.365 & {$[-0.060,0.294]$} & 0.174 \\
Interaction Effect (Int.): ER * GPR & & & \\
Int. $\longrightarrow$ PHR0.330 & 0.126 & 2.611 & {$[0.097,0.596]$} & 0.010 \\
& & & & \\
\hline
\end{tabular}

This significant result is supported by output presented in the "Index of Moderated Mediation" table (Table 4b) because the bootstrap CI does not include zero (Hayes, 2018).

TABLE 4A

CONDITIONAL INDIRECT EFFECT OF LOCAL PREDICTOR (ER) ON RPT AT VALUES OF THE MODERATOR (GPR)

\begin{tabular}{lccc}
\hline Description (Percentile) & Estimates & BootSE & $95 \%$ CI (bootstrap) \\
\hline $16^{\text {th }}$ percentile $(-0.544)$ & -0.011 & 0.029 & {$[-0.078,0.045]$} \\
$50^{\text {th }}$ percentile $(0.151)$ & 0.072 & 0.033 & {$[0.013,0.141]$} \\
$84^{\text {th }}$ percentile $(0.2251)$ & 0.810 & 0.037 & {$[0.015,0.159]$}
\end{tabular}

TABLE 4B

INDEX OF MODERATED MEDIATION

\begin{tabular}{lllc}
\hline & Estimates & \multicolumn{2}{l}{ BootSE 95\% CI (bootstrap) } \\
\hline $\begin{array}{l}\text { Indirect Effect } \\
\text { Moderator (GPR) }\end{array}$ & 0.119 & 0.065 & {$[0.014,0.262]$}
\end{tabular}

The effects found in our analysis are depicted with a graph, as shown in Figure 3 with Risk Perceptions for T2DM (RPT) as the dependent variable (y axis), Environment Risk (ER) as the independent variable (x axis) and General Public Risk (GPR) as the moderator. As depicted by the graph, at low levels of GPR, there is no statistically positive correlation between ER and RPT. However, as participants' GRP perceptions increase, so do their risk perceptions for acquiring T2DM. As a predictor of RPT, the ER x GPR interaction has a statistically significant effect. Specifically, when GPR is very low (that is, at the level of the 10th percentile), the indirect effect of ER is not significant. However, as the level of GPR increases, the indirect effect of ER on RPT increases in size until reaching statistical significance at the mean percentile (50th percentile) and higher percentile level (that is, 84th percentile). 
Thus, the variable of participants' risk perception for T2DM was significantly affected for participants who perceived highly general public risk factors (such as eating a healthy diet, having diabetes during pregnancy, or exercising regularly) and for those who perceived highly environmental risks (such as using illegal drugs and being exposed to medical x-rays, extreme hot or cold weather, air pollution, pesticides, household chemicals, and secondhand cigarette smoke).

FIGURE 3

\section{MODERATING EFFECT OF GPR ON THE RELATIONSHIP BETWEEN ER AND RPT}

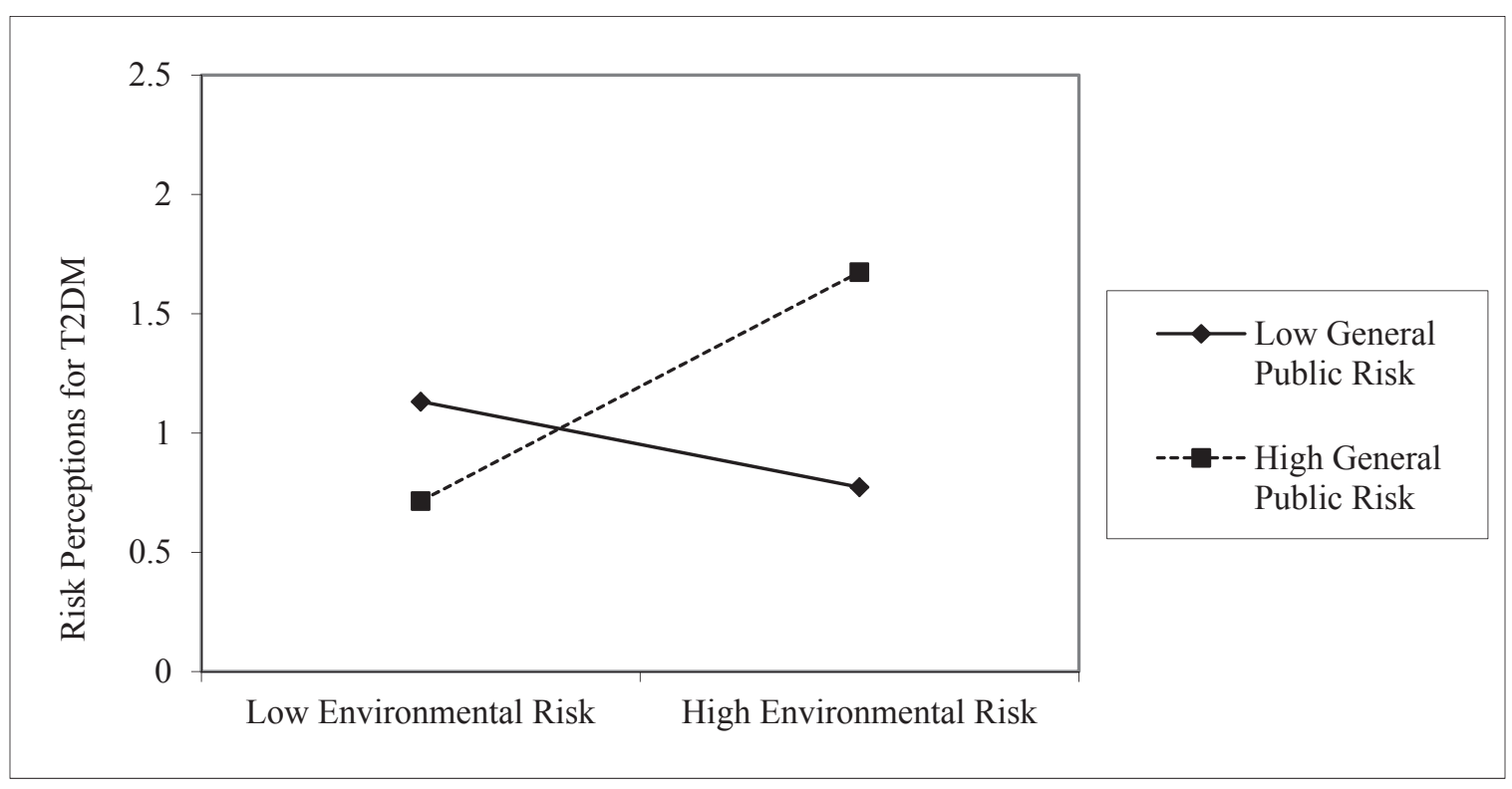

\section{DISCUSSION}

The findings of the data analysis provide support to our proposed model shown in Figure 1, which hypothesizes significant effects of multiple risk factors on students' risk perception of acquiring T2DM. Our model predicts that an individual's risk perception of acquiring T2DM is initially triggered by his or her interpretation of risk in the surrounding environment, particularly of the events that pose a potential threat to their well-being. The findings support this prediction indicating that some of the most commonly assessed threats representing moderate to high risk included illegal drugs, riding and/or driving a car, and secondhand cigarette smoke. The model also predicts that individuals develop a unique set of perceptions of risks related to their own personal health. Among the diseases surveyed in the sample, heart disease, cancer, and high blood pressure were perceived by the respondents to carry the highest risk. Finally, the model predicts that the relationship between individual's environmental (ER), personal (PR), and overall T2DM risk (RPT) perceptions is "activated" by their perceptions of the risks in the general public domain (GPR), which are operationalized by both demographic and lifestyle components.

Our findings indicate that students should be educated about the relative health consequences of acquiring T2DM, which include limb amputations, pregnancy complications, and cardiovascular diseases (Campbell, 2009). The risks leading to these health consequences should be included in course curricula to ensure greater awareness among students. Therefore, course curricula should be designed to increase the awareness of T2DM risk factors with an emphasis on T2DM's onset, prevention, and delay. The emphasis should be also placed on the related gender differences because females are not only more likely to have increased lifetime risk perceptions for developing T2DM than males but also are more likely to develop comparatively higher T2DM risk perceptions (Amuta et al., 2016). Moreover, academics should engage more in developing effective collaboration with health professionals, clinics, doctors, hospitals, 
and parents (Shaibi et al., 2015). The collaborative initiatives should focus on ensuring a sustainability of intervention measures and on efforts aimed at supporting college students' effort towards improving their dietary lifestyle during the age period when they experience lifestyle modifications. Furthermore, the use of appropriate apps can be very instrumental in this process.

\section{PRACTICAL IMPLICATIONS}

For administrators of higher education institutions, who plan to increase health awareness about T2DM and implement organizational policies and training programs aimed at educating students about the health risks related to T2DM, should collect and disseminate adequate and up-to-date data on the perceptions of T2DM among college students. To gather such data, more empirical research is required. Students currently living with T2DM can follow the practical step of regulating their sugar level by measuring the sugar level five times a day or one day per week (Mol, 2009). This instruction should be based on their awareness that regularly measuring diabetes may be challenging due to several factors such as inaccurate machine readings, lack of appropriate locations at work to measure the blood sugar level, stigma attached to having T2DM, and technical requirement reading and understanding the output from the diabetes machine.

Several relevant practical implications can be derived from our study's findings. First, our findings support those reported in the extant literature indicating that a large number of people, particularly young people such as students are unaware of T2DM risk factors. Regardless of whether the risk factors are modifiable are not, these are the factors that everyone, including students, should not only be aware of but also should take preventative measures to limit their effect on risk exposure to T2DM. Second, students should be informed duly about T2DMand the associate risks of acquiring it at an early age. General education on health consequences of acquiring T2DM, accompanied by instructions for using specific appropriate drugs and medical devices (Kahn, 2009) is necessary for managing diabetes effectively and planning, the related physical activities in physical education classes (Fischetti, 2015) because they are important tools to employ in the fight against T2DM. As Jacobson (2009) notes, "advances in technology have helped to improve outcomes and made treatment easier in some ways, yet more complex in others. For type 2 diabetes in particular, onset of disease means that long-standing dietary activity and activity habits must be altered, against a background of caloric overabundance and sedentary work and leisure time" (p. 1746). Third, healthcare professionals should make concerted efforts aimed at communicating to patients T2DM preventative measures and negative health effects. In addition, greater awareness of the role of diet and exercise, as well as regular testing of blood-glucose levels, may significantly strengthen the health and wellness of adolescents.

\section{LIMITATIONS AND CONCLUSIONS}

Our study has certain limitations. First, the sample size is limited to 155 students, although the sample is not unrepresentative of the general population of students living in the United States. This low sample size is however not uncommon in studies examining T2DM or perceptions of health related factors (such as obesity) (e.g. Årsand, Tatara, Østengen \& Hartvigsen, 2010; Perrin, Vann, Benjamin, Skinner, Wegner \& Ammerman, 2010; Fischetti, 2015). For example, Årsandet al. (2010) examined mobile phone-based self-management tools for type 2 diabetes of 12 participants while Perrin et al. (2010) studied 115 parents to determine whether accuracy of parental perception of children's weight status and reports of related behaviors changed following a brief pediatrics resident intervention. For future research, the authors recommend a sample closer to 200 participants (MacCullum, Widaman, Zhang, \& Hong, 1999). Second, some scale estimates proved unreliable. The unreliability of the instrument may be due to the homogeneity of the sample as study participants were mostly Caucasian (see also, Fishcetti, 2015). Future researchers should vary the sample to ensure higher psychometric validity while limiting effects of homogeneity of variance (Nunnally \& Bernstein, 1994). Third, due to the self-report nature of the study 
(Gorber, Tremblay, Moher, \& Gorber 2007; Prince et al., 2008), the results may be susceptible to recall bias and thus underestimate the perceptions that participants report (Amuta et al., 2016).

Despite these limitations, the study's findings contribute to the growing body of research on adolescent's risk perceptions of T2DM. With health trends rapidly declining and sedentary lifestyles taking over much of the world, it is more important than ever to increase the quality of education that young people are receiving to protect their well-being against the risk of acquiring T2DM. The authors agree with the recommendations of extant research (e.g. Weigensberg, 2009) that future researchers should address the impact of education and family-centered lifestyle on adolescents' perceptions of having T2DM. Moreover, it is in everyone's best interest to continue to strengthen the line of communication between health professionals and laypeople in order to curb the health epidemic that our world is currently facing. In conclusion, the following citation from Amuta, Barry, Popoola, and Crosslin (2015) succinctly illustrates this study's implications:

"In sum, young adults may not be aware of their susceptibility to T2DM as a result of an unhealthy lifestyle, misperceptions of risk, and gender differences. Therefore, ... health education programs are warranted to provide an opportunity to learn about lifestyle modifications early on in their lives." (p. 321)

\section{REFERENCES}

Alawneh, A., Yasin, H., Khirfan, G., Qayas, B. A., Ammar, K., Rimawi, D., \& Klepstad, P. (2016). Psychometric properties of the Arabic version of EORTC QLQ-C15-PAL among cancer patients in Jordan. Supportive Care in Cancer, 24(6), 2455-2462.

Alcolado, J. C., \& Alcolado, R. (1991). Importance of maternal history of non-insulin dependent diabetic patients. $B M J, 302(6786), 1178-1180$.

Amuta, A. O., Jacobs, W., Barry, A. E., Popoola, O. A., \& Crosslin, K. (2016). Gender differences in type 2 diabetes risk perception, attitude, and protective health behaviors: a study of overweight and obese college students. American Journal of Health Education, 47(5), 315-323.

Årsand, E., Tatara, N., Østengen, G., \& Hartvigsen, G. (2010). Mobile phone-based self-management tools for type 2 diabetes: The few touch application. Journal of Diabetes Science and Technology, 4(2), 328-336.

Bandura, A. (1977). Self-efficacy: toward a unifying theory of behavioral change. Psychological Review, 84(2), 191.

Bandura, A. (1986). Social foundations of thought and action: A social cognitive theory. Englewood Cliffs, NJ: Prentice Hall.

Bandura, A. (1991). Social cognitive theory of self-regulation. Organizational Behavior and Human Decision Processes, 50(2), 248-287.

Bandura, A. (1999). Moral disengagement in the preparation of inhumanities. Personal and Social Psychology Review, 3, 193-209.

Bloomgarden, Z. T. (2004). Type 2 diabetes in the young: the evolving epidemic. Diabetes Care, 27(4), 998-1010.

Campbell, D. (2009). Campbell: Better evidence for a better world. The Lancet, 373, 1735. DOI: https://doi.org/10.1016/S0140-6736(09)60955-7

Centers for Disease Control and Prevention (2017). National Diabetes Statistics Report. Atlanta, GA: Centers for Disease Control and Prevention, US Department of Health and Human Services.

Claassen, L., Henneman, L., Janssens, A. C. J., Wijdenes-Pijl, M., Qureshi, N., Walter, F. M., ... \& Timmermans, D. R. (2010). Using family history information to promote healthy lifestyles and prevent diseases; a discussion of the evidence. BMC Public Health, 10(1), 248.

D'adamo, E., \& Caprio, S. (2011). Type 2 diabetes in youth: epidemiology and pathophysiology. Diabetes Care, 34(Supplement 2), S161-S165. doi:10.2337/dc11-s212 
Fischetti, N. (2015). Perceived Risk for Developing Type 2 Diabetes in Adolescents. Pediatric Nursing Journal, 41(3), 126-131.

Font, X., Garay, L., \& Jones, S. (2016). A social cognitive theory of sustainability empathy. Annals of Tourism Research, 58, 65-80.

Geetha, A., Gopalakrishnan, S., \& Umadevi, R. (2017). Study on the impact of family history of diabetes among type 2 diabetes mellitus patients in an urban area of Kancheepuram district, Tamil Nadu. International Journal of Community Medicine and Public Health, 4(11), 4151-4156.

Godin, G. (2011). The Godin-Shephard leisure-time physical activity questionnaire. The Health \& Fitness Journal of Canada, 4(1), 18-22.

Gorber, S. C., Tremblay, M., Moher, D., \& Gorber, B. (2007). A comparison of direct vs. self-report measures for assessing height, weight and body mass index: a systematic review. Obesity Reviews, 8(4), 307-326.

Hayes, A. F. (2018). Introduction to mediation, moderation, and conditional process analysis: A regression-based approach (2nd edition). New York: The Guilford Press.

Homko, C. J., Zamora, L., Santamore, W. P., Kashem, A., McConnell, T., \& Bove, A. A. (2010). Gender differences in cardiovascular risk factors and risk perception among individuals with diabetes. The Diabetes Educator, 36(3), 483-488.

IBM Corp. Released 2017. IBM SPSS Statistics for Windows, Version 25.0. Armonk, NY: IBM Corp

Jacobson, A. M (2009). Diabetes: Finding "a clean well-lighted place." The Lancet, 373, 1746. DOI: https://doi.org/10.1016/S0140-6736(09)60963-6

Janz, N. K., \& Becker, M. H. (1984). The health belief model: A decade later. Health Education Quarterly, 11(1), 1-47

K Papazafiropoulou, A., Papanas, N., Melidonis, A., \& Maltezos, E. (2017). Family history of type 2 diabetes: does having a diabetic parent increase the risk? Current Diabetes Reviews, 13(1), 19-25.

Kahn, R. (2009). Diabetes technology—now and in the future. The Lancet, 373(9677), 1741-1743.

Kreps, T. A., \& Monin, B. (2011). Doing well by doing good? Ambivalent moral framing in organizations. Research in Organizational Behavior, 31, 99-123.

MacCallum, R. C., Widaman, K. F., Zhang, S., \& Hong, S. (1999). Sample size in factor analysis. Psychological Methods, 4(1), 84.

Mayo Clinic. (2017). Type 2 diabetes: Symptoms and causes. Retrieved September 15, 2018, from, https://www.mayoclinic.org/diseases-conditions/type-2-diabetes/symptoms-causes/syc-20351193

Mei, B., \& Brown, G. T. (2018). Conducting online surveys in China. Social Science Computer Review, 36(6), 721-734.

Mol, A. (2009). Living with diabetes: care beyond choice and control. The Lancet, 373(9677), 1756-1757.

National Institute of Diabetes and Digestive and Kidney Diseases. (2017). Type 2 Diabetes: What is type 2 diabetes? Retrieved January 23, 2019, from: https://www.niddk.nih.gov/health-information/ diabetes/overview/what-is-diabetes/type-2-diabetes

Nunnally, J.C., \& Bernstein, I.C. (1994). Psychometric theory (3rd ed.). New York, NY.

Perrin, E. M., Vann, J. C. J., Benjamin, J. T., Skinner, A. C., Wegner, S., \& Ammerman, A. S. (2010). Use of a pediatrician toolkit to address parental perception of children's weight status, nutrition, and activity behaviors. Academic Pediatrics, 10(4), 274-281.

Persons, O. (2009). Using a corporate code of ethics to assess students' ethicality: Implications for business education. Journal of Education for Business, 84(6), 357-366.

Popoola, I. T, Garner, B., Ammeter, A., Krey, N., Beu Ammeter, D., \& Schafer, S. (2017). How does ethics institutionalization reduce academic cheating? Journal of Education for Business, 92(1), 29-35

Preacher, K. J., Rucker, D. D., \& Hayes, A. F. (2007). Assessing moderated mediation hypotheses: Theory, methods, and prescriptions. Multivariate Behavioral Research, 42, 185-227.

Prince, S. A., Adamo, K. B., Hamel, M. E., Hardt, J., Gorber, S. C., \& Tremblay, M. (2008). A comparison of direct versus self-report measures for assessing physical activity in adults: a systematic review. International Journal of Behavioral Nutrition and Physical Activity, 5(1), 56. 
Rouyard, T., Kent, S., Baskerville, R., Leal, J., \& Gray, A. (2017). Perceptions of risks for diabetesrelated complications in Type 2 diabetes populations: a systematic review. Diabetic Medicine, 34(4), 467-477.

Sealey-Potts, C., \& Reyes-Velazquez, W. (2014). Perceived and actual risks of college students for developing type 2 diabetes. Austin Journal of Nutrition and Metabolism, 1(2), 1-5.

Shaibi, G. Q., Konopken, Y. P., Nagle-Williams, A., McClain, D. D., Castro, F. G., \& Keller, C. S. (2015). Diabetes Prevention for Latino Youth: Unraveling the Intervention "Black Box". Health Promotion Practice, 16(6), 916-924.

Valdez, R., Yoon, P. W., Liu, T., \& Khoury, M. J. (2007). Family history and prevalence of diabetes in the US population: The 6-year results from the National Health and Nutrition Examination Survey (NHANES, 1999-2004). Diabetes Care, 30(10), 2517-2522

Vornanen, M., Konttinen, H., Kääriäinen, H., Männistö, S., Salomaa, V., Perola, M., \& Haukkala, A. (2016). Family history and perceived risk of diabetes, cardiovascular disease, cancer, and depression. Preventive Medicine, 90, 177-183.

Walker, E. A., Caban, A., Schechter, C. B., Basch, C. E., Blanco, E., DeWitt, T., ... \& Mojica, G. (2007). Measuring comparative risk perceptions in an urban minority population. The Diabetes Educator, 33(1), 103-110.

Walker, E. A., Mertz, C. K., Kalten, M. R., \& Flynn, J. (2003). Risk perception for developing diabetes: comparative risk judgments of physicians. Diabetes Care, 26(9), 2543-2548.

Waters, E. A., McQueen, A., \& Cameron, L. D. (2013). 11 Perceived Risk and its Relationship to HealthRelated Decisions and Behavior. The Oxford Handbook of Health Communication, Behavior Change, and Treatment Adherence, 193.

Weigensberg, M (2009). Type 2 diabetes in children and adolescents. The Lancet, 373(9677), 1743-1744. 